\title{
Associations between eating frequency and energy intake, energy density, diet quality and body weight status in adults from the USA
}

\author{
Yong $\mathrm{Zhu}^{1}$ and James H. Hollis ${ }^{2 *}$ \\ ${ }^{1}$ Department of Epidemiology, The University of Iowa, Iowa City, IA 52242, USA \\ ${ }^{2}$ Department of Food Science and Human Nutrition, Iowa State University, Ames, IA 50011, USA \\ (Submitted 30 September 2015 - Final revision received 16 February 2016 - Accepted 29 February 2016 - First published online 25 April 2016)
}

\section{Abstract}

To investigate associations between eating frequency and energy intake, energy density, diet quality and body weight status in adults from the USA, combined data from the 2009-2010 and 2011-2012 National Health and Nutrition Examination Survey (NHANES) were used in this study. The first 24-h dietary recall data from eligible participants ( 4017 men and 3774 women) were used to calculate eating frequency, as well as energy intake, energy density and the Healthy Eating Index 2010 (HEI-2010), as a measure of diet quality. BMI and waist circumference were obtained from the NHANES body measures data. Adjusting for confounding socio-demographic characteristics and lifestyle factors, a higher eating frequency was significantly associated with higher energy intake in both men and women (both $P<0 \cdot 001)$. A higher eating frequency was also significantly associated with lower energy density in both men and women, regardless of whether beverage or water intake was included in the calculation of energy density (all $P<0 \cdot 01$ ). Moreover, there was a significant positive association between eating frequency and the HEI-2010 total score in both men and women (both $P<0 \cdot 001$ ). Eating frequency was inversely associated with BMI in women $(P=0.003)$, as well as waist circumference in both men $(P=0.032)$ and women $(P=0 \cdot 010)$. Results from the present study suggested that adults with a higher eating frequency in the USA had a healthier diet with lower energy density and better diet quality, and eating frequency was inversely associated with body weight status.

Key words: National Health and Nutrition Examination Survey: Eating frequency: Energy density: Diet quality

Owing to the recent and rapid rise in the number of overweight and obese people, it is thought that changing environmental factors such as dietary choices or lifestyle habits are the predominant cause for this epidemic. One potentially key environmental change that has occurred in concert with the obesity epidemic is an increase in eating frequency (the number of eating occasions) ${ }^{(1)}$. However, the association between eating frequency and energy intake, energy density, diet quality, BMI and waist circumference in a population-based nationally representative study has not been well studied.

Increasing eating frequency could possibly influence energy intake through improved appetite control. To date, the relationship between eating frequency and energy intake is not clear, with results reporting that increased eating frequency is associated with reduced food intake ${ }^{(2)}$, whereas another study found that increased eating frequency is associated with increased food intake ${ }^{(3)}$. It has also been suggested that eating frequency could affect diet-induced thermogenesis, although the results are inconsistent. Although a short-term $(<24 \mathrm{~h})$ study has suggested that increased eating frequency results in increased thermogenesis ${ }^{(4)}$, other studies have reported that increasing eating frequency decreases thermogenesis ${ }^{(5,6)}$ or has no effect $^{(7)}$. Longer-term studies (7d) using doubly labelled water have consistently reported no effects of eating frequency on energy expenditure ${ }^{(8,9)}$.

Similar to short-term studies of the components of energy balance, studies that have investigated the association between eating frequency and body weight status also provide inconsistent data. Epidemiological studies have reported an inverse association $^{(2,10,11)}$, no association ${ }^{(3,12)}$ or a positive association $^{(13)}$ between eating frequency and body weight status. These discrepant findings may be due to differences in the definition of eating frequency, differences in the measurement of eating frequency or the lack of adjustment for potential confounders $^{(14,15)}$. Only a limited number of randomisedcontrolled trials have been conducted thus far; they have reported that manipulating eating frequency had no effect on weight loss after intervention of 8 weeks ${ }^{(16)}$ or 6 months ${ }^{(17)}$. These studies focused on weight loss efficacy and were not representative of the wider population. Consequently, the results may be of limited relevance for efforts to optimise the diet for the general public. Further research, especially using

Abbreviations: HEI-2010, Healthy Eating Index 2010; NHANES, National Health and Nutrition Examination Survey.

* Corresponding author: J. H. Hollis, email jhollis@iastate.edu 
nationally representative data, is warranted to investigate the relationship between eating frequency and diet and body weight status.

\section{Methods \\ Data source and study population}

Data from the 2009-2010 and 2011-2012 National Health and Nutrition Examination Survey (NHANES) were combined and used in this study. The NHANES is a survey conducted by the National Center for Health Statistics for the assessment of health and nutritional status of non-institutionalised residents in the USA; selected participants were invited for the interview and physical examinations ${ }^{(18)}$. At the mobile examination centre, participants were asked to provide a 24-h dietary recall; a second dietary recall was obtained by telephone interview 3-10 d later. The NHANES dietary recall was administered using the United States Department of Agriculture (USDA) Automated Multiple-Pass Method ${ }^{(19)}$, and participants were provided with a standard set of measuring guides to help estimate food portion sizes. Dietary recall data were processed using the USDA's Food and Nutrient Database for Dietary Studies; details of sampling methodology, survey instruments, study protocols and data sets are available on the NHANES website ${ }^{(18)}$. The NHANES study was conducted according to the guidelines laid down in the Declaration of Helsinki, and all procedures involving human subjects were approved by the National Center for Health Statistics Research Ethics Review Board. Written informed consent was obtained from all the participants.

Participants aged $\geq 20$ years with a complete first 24-h dietary recall were initially included in this study ( $n$ 10563). Of these, 2772 participants were excluded if they met one or more of the following criteria: were pregnant or lactating (168 participants), were on a special diet either to lose weight or for some other health-related reason (1520 participants) or had missing data on any covariates or outcome variables (1310 participants). The final sample size of the present study included 7791 eligible adults (4017 men and 3774 women).

\section{Exposure and outcome variables}

Self-reported time of consumption of each food or beverage in response to the question 'what time did you begin to eat/drink the meal/food?' in the first $24-\mathrm{h}$ dietary recall was used to estimate eating frequency. An eating occasion was defined such that it contained at least $210 \mathrm{~kJ}$ and the time interval between eating occasions was more than $15 \mathrm{~min}^{(20)}$. This definition was chosen because recent studies that examined various definitions of eating occasion revealed it was the one that best predicted variance in total energy intake ${ }^{(21)}$ or provided the strongest correlation with total energy intake ${ }^{(22)}$

Total energy intake from the first dietary recall, BMI and waist circumference were directly obtained from the NHANES data set. Energy density was calculated by dividing total energy of dietary items by total weight of dietary items ${ }^{(23-25)}$. Three types of energy density were examined in this study: using foods only, using foods and beverages excluding water and using foods and beverages including water. Milk and milk drinks, fruit juice, vegetable juice, alcoholic beverages and non-alcoholic beverages (coffee, tea, soft drinks, etc.) were considered as beverages ${ }^{(25)}$

Diet quality was estimated as the Healthy Eating Index 2010 $(\text { HEI-2010 })^{(26)}$. The HEI-2010 was developed as a parameter for compliance with the 2010 Dietary Guidelines for Americans; it consists of nine adequacy food components and three moderation food components; the total score ranges from 0 to 100, a higher score indicates better diet quality or better compliance with the dietary guidelines ${ }^{(26)}$.

\section{Covariates}

Socio-demographic characteristics including age, race/ethnicity and ratio of family income:poverty, as well as lifestyle factors such as physical activity level and smoking status, were used as covariates. Classifications of these variables were the same as the approach used in a previous study ${ }^{(27)}$. In brief, race/ethnicity was classified as Hispanic, non-Hispanic white, non-Hispanic black or other; ratio of family income:poverty was coded to a categorical variable as $\leq 1.85,1 \cdot 86-3.50,>3.50$; physical activity level was classified as vigorously active, moderately active or sedentary, which was estimated from answers to questions 'does your work involve vigorous-intensity activity that causes large increases in breathing or heart rate for at least $10 \mathrm{~min}$ continuously' and 'do you do any moderate-intensity sports, fitness or recreational activities that cause a small increase in breathing or heart rate for at least $10 \mathrm{~min}$ continuously'; and smoking status was coded as non-smoker, former smoker or current smoker. Moreover, total energy intake was also adjusted for in the analysis where appropriate.

\section{Statistical analysis}

SAS version 9.3 (SAS Institute) was used for statistical analysis; 4 -year sample weight and specific SAS survey procedures were used to account for the complex multistage design of the NHANES. As a previous study reported sex-specific associations between eating frequency and cardiometabolic risk factors ${ }^{(11)}$, data for men and women were analysed separately in the main analyses to examine potential sex-specific differences. Characteristics of participants were compared between sexes by survey $\chi^{2}$ tests for categorical variables and by survey linear regression for continuous variables. Survey multivariate linear regression was used to estimate covariate-adjusted associations between eating frequency and each outcome of interests. Data were presented as weighted percentages, least squares means or regression coefficients with their standard errors, where appropriate. The statistical significance was considered as $P<0.05$.

Two sensitivity analyses were conducted to determine associations between eating frequency and total energy intake, energy density, the HEI-2010 total score, BMI and waist circumference. First, a pooled analysis combining data from men and women was conducted, with sex as an additional covariate in the statistical model. Second, the associations were analysed using the second 24-h dietary recall data from participants whose complete data were available. 


\section{Results}

\section{Characteristics of the participants}

The average eating frequency was 4.48 ( $\mathrm{se} 0 \cdot 03$, range $1-10)$ in men and 4.38 (se 0.03, range 1-12) in women. Characteristics of the participants (men and women) are presented in Table 1. Significant sex differences were found in race/ethnicity, ratio of family income:poverty, physical activity, smoking status and age $(P<0.05$ for all). The difference in BMI was not significant between men and women $(P=0.430)$; however, men had a significantly greater waist circumference than women $(P<0 \cdot 001)$. Men also had significantly higher total energy intake and higher energy density (except when water was not included in the calculation of energy density), as well as a significantly lower HEI-2015 total score, compared with women ( $P<0.05$ for all). Examination of individual component scores of the HEI-2015 revealed that men were less compliant to the dietary guidelines compared with women for most types of foods (Table 1).

\section{Energy intake and energy density}

There was a significant positive association between eating frequency and total energy intake in both men and women (both $P<0.001$, Table 2). A significant inverse association between eating frequency and energy density was found in

Table 1. Characteristics of the participants

(Weighted percentages, least squares means with their standard errors)

\begin{tabular}{|c|c|c|c|c|c|c|c|}
\hline \multirow[b]{2}{*}{ Categorical variables } & \multicolumn{3}{|c|}{ Men $(n$ 4017) } & \multicolumn{3}{|c|}{ Women ( $n$ 3774) } & \multirow[b]{2}{*}{$P^{*}$} \\
\hline & $n$ & Weighted \% & SE & $n$ & Weighted \% & SE & \\
\hline Race/ethnicity & & & & & & & 0.002 \\
\hline Hispanic & 941 & $14 \cdot 0$ & $2 \cdot 0$ & 896 & $12 \cdot 7$ & 1.8 & \\
\hline Non-Hispanic white & 1839 & 69.9 & 2.5 & 1682 & 68.4 & $2 \cdot 6$ & \\
\hline Non-Hispanic black & 828 & $9 \cdot 6$ & 1.1 & 814 & 11.7 & 1.3 & \\
\hline Other & 409 & 6.5 & 0.8 & 382 & $7 \cdot 1$ & 0.8 & \\
\hline Ratio of family income:poverty & & & & & & & $<0.001$ \\
\hline$\leq 1.85$ & 1835 & 31.8 & 1.6 & 1892 & $36 \cdot 2$ & 1.4 & \\
\hline $1.86-3.50$ & 910 & $23 \cdot 9$ & $1 \cdot 1$ & 856 & $25 \cdot 6$ & 1.3 & \\
\hline$>3.50$ & 1272 & 44.3 & 1.6 & 1026 & $38 \cdot 3$ & $2 \cdot 0$ & \\
\hline Physical activity & & & & & & & $<0.001$ \\
\hline Vigorously active & 1786 & 51.0 & 1.4 & 910 & $28 \cdot 3$ & 1.4 & \\
\hline Moderately active & 1149 & $27 \cdot 7$ & $1 \cdot 1$ & 1397 & $38 \cdot 2$ & 1.1 & \\
\hline Sedentary & 1082 & $21 \cdot 3$ & 1.0 & 1467 & 33.5 & 1.2 & \\
\hline Smoking status & & & & & & & $<0.001$ \\
\hline Non-smoker & 1839 & $49 \cdot 1$ & 1.5 & 2377 & $60 \cdot 2$ & $1 \cdot 1$ & \\
\hline Former smoker & 1136 & $27 \cdot 4$ & 1.2 & 669 & $20 \cdot 2$ & 1.2 & \\
\hline Current smoker & 1042 & 23.5 & $1 \cdot 1$ & 728 & $19 \cdot 6$ & $1 \cdot 0$ & \\
\hline Continuous variables & & $\begin{array}{l}\text { Least squares } \\
\text { mean }\end{array}$ & SE & & $\begin{array}{l}\text { Least squares } \\
\text { mean }\end{array}$ & SE & \\
\hline Age (years) & & $46 \cdot 1$ & 0.5 & & 47.4 & 0.5 & $<0.001$ \\
\hline BMI $\left(\mathrm{kg} / \mathrm{m}^{2}\right)$ & & $28 \cdot 4$ & 0.2 & & $28 \cdot 2$ & 0.2 & 0.430 \\
\hline Waist circumference $(\mathrm{cm})$ & & $100 \cdot 4$ & 0.5 & & 94.6 & 0.5 & $<0.001$ \\
\hline Eating occasions & & 4.48 & 0.03 & & 4.38 & 0.03 & 0.005 \\
\hline Energy intake (kJ) & & 10873 & 96 & & 7706 & 52 & $<0.001$ \\
\hline Energy density $(\mathrm{kJ} / \mathrm{g}) \dagger$ & & 8.38 & 0.06 & & 7.92 & 0.07 & $<0.001$ \\
\hline Energy density $(\mathrm{kJ} / \mathrm{g}) \ddagger$ & & $4 \cdot 16$ & 0.04 & & 4.09 & 0.04 & 0.107 \\
\hline Energy density $(\mathrm{kJ} / \mathrm{g}) \S$ & & 2.39 & 0.03 & & $2 \cdot 29$ & 0.02 & 0.006 \\
\hline HEI-2010 total score & & $48 \cdot 24$ & 0.39 & & 49.88 & 0.47 & $<0.001$ \\
\hline $\mathrm{HEl}-2010$ total vegetables score & & $2 \cdot 68$ & 0.03 & & 2.96 & 0.04 & $<0.001$ \\
\hline HEI-2010 greens and beans score & & 1.06 & 0.05 & & 1.30 & 0.06 & 0.001 \\
\hline HEI-2010 total fruit score & & 2.00 & 0.04 & & 2.35 & 0.06 & $<0.001$ \\
\hline HEI-2010 whole fruit score & & 1.93 & 0.05 & & $2 \cdot 28$ & 0.06 & $<0.001$ \\
\hline HEI-2010 whole grains score & & 1.97 & 0.09 & & 2.37 & 0.08 & $<0.001$ \\
\hline HEI-2010 dairy products score & & $4 \cdot 20$ & 0.08 & & 4.69 & 0.08 & $<0.001$ \\
\hline HEI-2010 total protein foods score & & 4.04 & 0.04 & & 3.76 & 0.04 & $<0.001$ \\
\hline HEI-2010 seafood and plant proteins score & & 1.94 & 0.05 & & 2.03 & 0.05 & 0.249 \\
\hline HEI-2010 fatty acids score & & 5.09 & 0.08 & & 5.09 & 0.09 & 0.987 \\
\hline HEI-2010 Na score & & 4.30 & 0.06 & & 4.36 & 0.08 & 0.541 \\
\hline HEI-2010 refined grains score & & 6.56 & 0.09 & & $6 \cdot 23$ & 0.07 & 0.001 \\
\hline $\begin{array}{l}\mathrm{HEI}-2010 \text { solid fat, alcohol and added sugar energy } \\
\text { content score }\end{array}$ & & $12 \cdot 47$ & $0 \cdot 18$ & & $12 \cdot 47$ & $0 \cdot 17$ & 0.976 \\
\hline
\end{tabular}

HEI-2010, Healthy Eating Index 2010.

* $P$ value for comparisons between men and women.

† Energy density calculated as energy from all food items divided by weight of all food items.

$\ddagger$ Energy density calculated as energy from all food items and beverages excluding water divided by weight of all food items and beverages excluding water.

$\S$ Energy density calculated as energy from all food items and beverages including water divided by weight of all food items and beverages including water. 
Table 2. Associations between eating frequency and energy intake and energy density (Regression coefficients with their standard errors)

\begin{tabular}{|c|c|c|c|c|c|c|}
\hline & \multicolumn{3}{|c|}{ Men ( $n$ 4017) } & \multicolumn{3}{|c|}{ Women ( $n$ 3774) } \\
\hline & $\beta$ & SE & $P$ & $\beta$ & SE & $P$ \\
\hline Energy intake $(\mathrm{kJ})^{*}$ & $264 \cdot 7$ & $18 \cdot 9$ & $<0.001$ & $204 \cdot 4$ & $9 \cdot 6$ & $<0.001$ \\
\hline Energy density (kJ/g)†‡ & $-0 \cdot 152$ & 0.037 & $<0.001$ & -0.218 & 0.034 & $<0.001$ \\
\hline Energy density $(\mathrm{kJ} / \mathrm{g})+\S$ & $-0 \cdot 105$ & 0.022 & $<0.001$ & -0.086 & 0.022 & $<0.001$ \\
\hline Energy density $(\mathrm{kJ} / \mathrm{g}) \dagger \|$ & -0.057 & 0.017 & 0.002 & -0.092 & 0.019 & $<0.001$ \\
\hline
\end{tabular}

* Adjusted for age, race/ethnicity, ratio of family income:poverty, physical activity level and smoking status; $\beta$ is the change in the outcome variable for each unit increase of eating frequency when adjusting for covariates.

† Adjusted for age, race/ethnicity, ratio of family income:poverty, physical activity level, smoking status and energy intake.

$\ddagger$ Energy density calculated as energy from all food items divided by weight of all food items.

$\S$ Energy density calculated as energy from all food items and beverages excluding water divided by weight of all food items and beverages excluding water.

\| Energy density calculated as energy from all food items and beverages including water divided by weight of all food items and beverages including water.

Table 3. Associations between eating frequency and the Health Eating Index 2010 (HEl-2010)* (Regression coefficients with their standard errors)

\begin{tabular}{|c|c|c|c|c|c|c|}
\hline & \multicolumn{3}{|c|}{ Men ( $n$ 4017) } & \multicolumn{3}{|c|}{ Women $(n$ 3774) } \\
\hline & $\beta$ & $\mathrm{SE}$ & $P$ & $\beta$ & $\mathrm{SE}$ & $P$ \\
\hline HEI-2010 total score & 1.698 & 0.243 & $<0.001$ & 1.630 & 0.245 & $<0.001$ \\
\hline \multicolumn{7}{|l|}{ Adequacy component } \\
\hline Total vegetables & -0.014 & 0.029 & 0.638 & 0.013 & 0.035 & 0.717 \\
\hline Greens and beans & -0.005 & 0.037 & 0.901 & 0.050 & 0.032 & 0.132 \\
\hline Total fruit & 0.332 & 0.030 & $<0.001$ & 0.328 & 0.031 & $<0.001$ \\
\hline Whole fruit & 0.341 & 0.032 & $<0.001$ & 0.366 & 0.031 & $<0.001$ \\
\hline Whole grains & 0.259 & 0.054 & $<0.001$ & 0.275 & 0.056 & $<0.001$ \\
\hline Dairy products & 0.243 & 0.060 & $<0.001$ & 0.252 & 0.052 & $<0.001$ \\
\hline Total protein foods & 0.025 & 0.030 & 0.398 & -0.044 & 0.034 & 0.199 \\
\hline Seafood and plant proteins & $0 \cdot 180$ & 0.040 & $<0.001$ & 0.110 & 0.038 & 0.006 \\
\hline Fatty acids & -0.091 & 0.063 & 0.159 & -0.119 & 0.056 & 0.040 \\
\hline \multicolumn{7}{|l|}{ Moderation component } \\
\hline $\mathrm{Na}$ & 0.217 & 0.065 & 0.002 & 0.275 & 0.054 & $<0.001$ \\
\hline Refined grains & 0.177 & 0.067 & 0.012 & 0.129 & 0.076 & 0.099 \\
\hline Solid fat, alcohol, and added sugar energy content & 0.033 & $0 \cdot 107$ & 0.759 & -0.005 & 0.112 & 0.963 \\
\hline
\end{tabular}

both men and women; this was observed regardless of inclusion of water and beverages in the calculation of energy density $(P<0.01$ for all, Table 2$)$.

\section{Diet quality}

Associations between eating frequency and the HEI-2010 total score and each component score are shown in Table 3. A significant positive association between eating frequency and diet quality was observed in both men and women (both $P<0.001$ ). Significant positive associations were also observed for most adequacy component scores, including total fruit, whole fruit, whole grains, dairy products and seafood and plant proteins, in both men and women $(P<0.01$ for all). In contrast, a significant negative association was observed between eating frequency and the fatty acids component score in women $(P=0.040)$ but not in men $(P=0 \cdot 159)$. For moderation components, the $\mathrm{Na}$ component score was significantly and positively associated with eating frequency in both men $(P=0.002)$ and women $(P<0.001)$, whereas the component score for refined grains was significantly and positively associated with eating frequency in men $(P=0 \cdot 012)$ but not in women $(P=0.099)$.

\section{$B M I$ and waist circumference}

As shown in Table 4, eating frequency was significantly and inversely associated with BMI in women $(P=0 \cdot 003)$; however, the association was not statistically significant in men $(P=0 \cdot 130)$. In contrast, eating frequency was significantly and inversely associated with waist circumference in both men $(P=0.032)$ and women $(P=0 \cdot 010)$.

\section{Sensitivity analysis}

The pooled analysis combining data from both men and women revealed that eating frequency was significantly and positively associated with energy intake, as well as significantly and inversely associated with energy density, diet quality, BMI and waist circumference ( $P<0.05$ for all, data not shown). Among participants included in the present study, 3470 men and 3420 women also provided a complete second 24-h 
Table 4. Associations between eating frequency and BMI and waist circumference* (Regression coefficients with their standard errors)

\begin{tabular}{|c|c|c|c|c|c|c|}
\hline & \multicolumn{3}{|c|}{ Men ( $n$ 4017) } & \multicolumn{3}{|c|}{ Women ( $n$ 3774) } \\
\hline & $\beta$ & SE & $P$ & $\beta$ & SE & $P$ \\
\hline BMI $\left(\mathrm{kg} / \mathrm{m}^{2}\right)$ & -0.166 & 0.107 & 0.130 & -0.400 & 0.123 & 0.003 \\
\hline Waist circumference $(\mathrm{cm})$ & -0.568 & 0.254 & 0.032 & -0.798 & 0.292 & 0.010 \\
\hline
\end{tabular}

dietary recall. The mean difference in eating frequency between the day of the first dietary recall and the day of the second dietary recall was 0.18 ( $\mathrm{SE} 0.03$ ) in men and 0.11 (SE 0.03) in women, respectively. Both differences were statistically significant $(P<0.001)$. Only $25.5 \%$ of men and $30.4 \%$ of women had the same eating frequency on both days; however, the difference in eating frequency between the two dietary recalls was no more than one eating occasion for the majority of participants $(67.9 \%$ of men and $72.1 \%$ of women). Analysis of the second 24-h dietary recall data also revealed similar results regarding associations between eating frequency and energy intake, energy density, diet quality and waist circumference $(P<0.05$ for all, data not shown), whereas the inverse associations between eating frequency and BMI in both men and women were statistically significant $(P=0.040$ and $P=0.003$, data not shown).

\section{Discussion}

There has been an increase in eating frequency over the past 30 years in all age groups ${ }^{(1)}$. The present study using nationally representative data from the USA found that men ate, on average, 4.48 times each day, whereas it was 4.38 times for women. A better understanding of how eating frequency influences body weight may lead to improved strategies to aid weight loss. Data from the present study suggest that increased eating frequency is associated with increased energy intake, a diet with lower energy density and better diet quality; moreover, it was inversely associated with BMI and waist circumference, although the association with BMI was not significant in men when the first 24-h dietary recall data were analysed.

Results from this study are consistent with previous results, which report a positive association between eating frequency and energy intake ${ }^{(3)}$, and several cross-sectional studies, which have found an inverse significant relationship between eating frequency and body weight or $\mathrm{BMI}^{(2,10)}$, but not others ${ }^{(3,12,13)}$. There are several possible explanations for these discrepant and paradoxical results. First, eating frequency and anthropometric measures are related to several socio-economic and lifestyle variables, which may confound the results. If these are not adequately controlled for, then spurious associations may be reported. The list of covariates adjusted for was not consistent among studies. Second, the true relationship between eating frequency and body weight may be masked by under-reporting of energy intake, which is an issue in common observational studies that rely on self-reported diet recalls ${ }^{(28)}$. Although randomised-controlled trials are better placed to show a causal relationship between eating frequency and anthropometric measures of interest, a few studies have been conducted thus far $^{(16,17)}$. These studies have altered eating frequency as part of an energy-restricted diet and generally show no effect of eating frequency on weight loss ${ }^{(16,17)}$. However, the results from these weight loss studies cannot be extrapolated to the general population who are not on a weight loss diet and wish to maintain a healthy body weight or prevent weight gain. Moreover, there are some weaknesses to randomised-controlled trials that could also mask a true relationship (e.g. poor participant compliance to the treatment protocol). Mechanistic data that may support a role for eating frequency also provide inconsistent results. One hypothesis is that increasing eating frequency may aid weight management through an effect on appetite control $^{(29)}$. However, no compelling evidence for this effect currently exists. Another plausible hypothesis is that increasing eating frequency increases dietary-induced thermogenesis. Currently available data also fail to support this hypothesis ${ }^{(5-7)}$. Owing to the inconsistent results and relative paucity of data, further research is required to clarify the role of eating frequency on weight management.

Several studies have found that a diet with higher energy density is associated with poor appetite control ${ }^{(30)}$ and a higher body weight ${ }^{(31,32)}$. However, the present study is not able to demonstrate a causal relationship between eating frequency and energy density. It is possible that individuals who eat more frequently are also more health conscious and choose lowenergy density foods. It is also possible that low-energy density foods influence eating frequency by a more rapid return of hunger, which stimulates an eating occasion ${ }^{(33)}$. Further research is warranted to understand how food characteristics influence eating patterns and how this influences health.

The present study provides evidence that increased eating frequency is associated with improved diet quality. As eating frequency increased, there was an increase in the HEI-2010 total score and higher component scores such as total fruits, whole fruits, whole grains, dairy products and seafood and plant proteins. One potential explanation for associations between eating frequency and diet quality is that people who are more health conscious eat healthier meals or snacks more frequently. A causal effect, however, remains to be investigated.

There are some limitations to the present study. First, the results do not indicate any causal relationship because the data were from a cross-sectional study. Second, the NHANES dietary data analysed may not reflect a dietary pattern over a longer 
period as there could be inter-day variations in dietary intake. As shown by a sensitivity analysis in the present study, although most results were similar as the main analysis, the associations between eating frequency and BMI in men were different depending on the day of the dietary recall. Moreover, the physical activity level included in the statistical model was derived from a self-reported questionnaire; this might not have completely ruled out the possibility of confounding. Finally, the present study did not aim to investigate the impact of snacking; therefore, eating frequency did not differentiate eating occasion of main meals from snacks. Nonetheless, to our knowledge, it was the first study that examined associations between eating frequency and diet quality using a nationally representative data. Longitudinal studies are encouraged to examine the chronic impact of a dietary pattern characterised by increased eating frequency on change in body weight.

\section{Acknowledgements}

The authors thank participants and investigators of the NHANES who made the data available.

This research received no specific grant from any funding agency, commercial or not-for-profit sectors.

Y. Z. and J. H. H. designed the present study, carried out the data analysis and prepared the manuscript.

The authors have no financial or personal conflicts of interest to declare.

\section{References}

1. Popkin BM \& Duffey KJ (2010) Does hunger and satiety drive eating anymore? Increasing eating occasions and decreasing time between eating occasions in the United States. Am J Clin Nutr 91, 1342-1347.

2. Aljuraiban GS, Chan Q, Griep LMO, et al. (2015) The impact of eating frequency and time of intake on nutrient quality and body mass index: the INTERMAP study, a populationbased study. J Acad Nutr Diet 115, 528-536.

3. Mills JP, Perry CD \& Reicks M (2011) Eating frequency is associated with energy intake but not obesity in midlife women. Obesity (Silver Spring) 19, 552-559.

4. Leblanc J, Mercier I \& Nadeau A (1993) Components of postprandial thermogenesis in relation to meal frequency in humans. Can J Physiol Pharmacol 71, 879-883.

5. Tai MM, Castillo P \& Pisunyer FX (1991) Meal size and frequency: effect on the thermal effect of food. Am J Clin Nutr 54, 783-787.

6. Westerterp-Plantenga MS, Goris AH, Meijer EP, et al. (2003) Habitual meal frequency in relation to resting and activityinduced energy expenditure in human subjects: the role of fat-free mass. Br J Nutr 90, 643-649.

7. Belko AZ \& Barbieri TF (1987) Effect of meal size and frequency on the thermal effect of food. Nutr Res 7, 237-242.

8. Verboeket-van de Venne WP, Westerterp KR \& Kester AD (1993) Effect of the pattern of food-intake on human energy metabolism. Br J Nutr 70, 103-115.

9. Verboeket-van de Venne WP \& Westerterp KR (1991) Influence of the feeding frequency on nutrient utilization in man: consequences for energy metabolism. Eur J Clin Nutr 45, 161-169.
10. Franko DL, Striegel-Moore RH, Thompson D, et al. (2008) The relationship between meal frequency and body mass index in black and white adolescent girls: more is less. Int $J$ Obes (Lond) 32, 23-29.

11. Smith KJ, Blizzard L, McNaughton SA, et al. (2012) Daily eating frequency and cardiometabolic risk factors in young Australian adults: cross-sectional analyses. Br J Nutr 108, 1086-1094

12. Hartline-Grafton HL, Rose D, Johnson CC, et al. (2010) The influence of weekday eating pattern on energy intake and BMI among female elementary school personnel. Obesity (Silver Spring) 18, 736-742.

13. Howarth NC, Huang TTK, Roberts SB, et al. (2007) Eating patterns and dietary composition in relation to BMI in younger and older adults. Int J Obes (Lond) 31, 675-684.

14. Bellisle F, McDevitt R \& Prentice AM (1997) Meal frequency and energy balance. Br J Nutr 77, S57-S70.

15. Kant AK (2014) Evidence for efficacy and effectiveness of changes in eating frequency for body weight management. Adv Nutr 5, 822-828.

16. Cameron JD, Cyr MJ \& Doucet E (2010) Increased meal frequency does not promote greater weight loss in subjects who were prescribed an 8-week equi-energetic energyrestricted diet. Br J Nutr 103, 1098-1101.

17. Bachman JL \& Raynor HA (2012) Effects of manipulating eating frequency during a behavioral weight loss intervention: a pilot randomized controlled trial. Obesity (Silver Spring) 20, 985-992.

18. Centers for Disease Control and Prevention (2014) National Health and Nutrition Examination Survey. http://www.cdc. gov/nchs/nhanes.htm (accessed November 2014).

19. Blanton CA, Moshfegh AJ, Baer DJ, et al. (2006) The USDA Automated Multiple-Pass Method accurately estimates group total energy and nutrient intake. J Nutr 136, 2594-2599.

20. Gibney MJ \& Wolever TMS (1997) Periodicity of eating and human health: present perspective and future directions. Br J Nutr 77, S3-S5.

21. Leech RM, Worsley A, Timperio A, et al. (2015) Characterizing eating patterns: a comparison of eating occasion definitions. Am J Clin Nutr 102, 1229-1237.

22. Murakami K \& Livingstone MBE (2014) Eating frequency in relation to body mass index and waist circumference in British adults. Int J Obes (Lond) 38, 1200-1206.

23. Kant AK \& Graubard BI (2005) Energy density of diets reported by American adults: association with food group intake, nutrient intake, and body weight. Int J Obes (Lond) 29 , 950-956.

24. Vernarelli JA, Mitchell DC, Rolls BJ, et al. (2013) Methods for calculating dietary energy density in a nationally representative sample. Procedia Food Sci 2, 68-74.

25. Zhu Y \& Hollis JH (2014) Soup consumption is associated with a lower dietary energy density and a better diet quality in US adults. Br J Nutr 111, 1474-1480.

26. Guenther PM, Casavale KO, Reedy J, et al. (2013) Update of the Healthy Eating Index: HEI-2010. J Acad Nutr Diet 113, 569-580.

27. Zhu Y \& Hollis JH (2014) Tooth loss and its association with dietary intake and diet quality in American adults. J Dent $\mathbf{4 2}$, 1428-1435.

28. Flegal KM (1999) Evaluating epidemiologic evidence of the effects of food and nutrient exposures. Am J Clin Nutr 69 , 1339S-1344S.

29. Leidy HJ \& Campbell WW (2011) The effect of eating frequency on appetite control and food intake: brief synopsis of controlled feeding studies. J Nutr 141, 154-157. 
30. Latner JD, Rosewall JK \& Chisholm AM (2008) Energy density effects on food intake, appetite ratings, and loss of control in women with binge eating disorder and weight-matched controls. Eat Behav 9, 257-266.

31. Vernarelli JA, Mitchell DC, Hartman TJ, et al. (2011) Dietary energy density is associated with body weight status and vegetable intake in US children. J Nutr 141, 2204-2210.
32. Vernarelli JA, Mitchell DC, Rolls BJ, et al. (2015) Dietary energy density is associated with obesity and other biomarkers of chronic disease in US adults. Eur J Nutr $\mathbf{5 4}$ 59-65.

33. Poppitt SD \& Prentice AM (1996) Energy density and its role in the control of food intake: evidence from metabolic and community studies. Appetite 26, 153-174. 